\title{
Effect of Treadmill training on Gait in Multiple Sclerosis patient: A randomized
} controlled trial

\author{
Moshira H. Darwish ${ }^{1}$, PhD; Sandra M. Ahmed ${ }^{2}$, PhD; Mahmoud Y. El Zanaty ${ }^{1}$, PhD \\ and Mohamed T. Emam'.
}

${ }^{1}$ Department of Physical Therapy for Neuromuscular Disorders, Faculty of Physical therapy, Cairo University.

${ }^{2}$ Department of Neurology, Faculty of Medicine, Cairo University, Egypt. Received: 05 Mar. 2019/ Accepted 14 April /2019 Publication date: 30 Dec. 2019

\begin{abstract}
Background and Objective: The most effective treatment approach to improve walking in people with multiple sclerosis (MS) is not known. The aim of this trial was to Evaluate the effect of treadmill training on gait in people with multiple sclerosis. Design: Randomized controlled trial. Setting: Multiple Sclerosis Center, Alkasr Al-Ainy Subjects: Forty people from both sex with multiple sclerosis, mean Expanded Disability Status Scale (S.D) was 4.3 (1.3). Their Age ranged from 25-45 years old, Last attack occured at least "three" months ago, Duration of illness started " one" to "two" years ago, Duration of immune regulatory therapy " one" to "two" years. Interventions: Participants were randomly allocated into two groups, study group(GA) received 8 week training sessions of physical therapy program in addition to treadmill training $(n=20)$. control group(GB) received physical therapy program $(n=20)$ in an outpatient basis. Main measures: Spatio-temporal parameters of walking which include step length and speed. Results: At the termination, there was significant increase $(\mathrm{p}<0.05)$ in left and right step length and speed in the post treatment condition compared with the pre treatment in both groups. Regarding between subject effects multiple pairwise comparisons revealed that there was significant increase $(p<0.05)$ in speed in favor to group A than group B. while there were no significant differences $(p>0.05)$ between both groups in left and right step length. Conclusions: Treadmill training in addition to physical therapy program are a possible treatment option for people with multiple sclerosis in order to improve their walking capabilities. However, this approach does not have any significant advantage over physical therapy program alone.
\end{abstract}

Keywords: Multiple sclerosis, gait, physical therapy

\section{Introduction}

Gait deficits are common in people with multiple sclerosis. These disabling deficits reduce mobility, independence, lead to falls and injuries and negatively affect quality of life. Generally, physical interventions directed at improving walking abilities have implemented various approaches, e.g. motor and sensory strategies, robot assisted gait training, sensory insoles, TAi-Chi exercises, Nintendo Wii games strength and aerobic training and neuromuscular facilitation. A popular alternative rehabilitation method is treadmill training in addition to physical therapy program. This program is a precise, controlled form of exercises which include stretching exercises, facilitation of weak muscles, strengthening exercises, proprioceptive neuro muscular facilitation, pelvic tilting exercises ,pelvic control exercises and gait training on treadmill (Cameron \& Wagner, 2011). This exercises can also be performed at various intensity levels whereby the participant or patient may adjust the difficulty to their own level of conditioning.

Despite a lack of scientific evidence supporting the effectiveness of these exercise training in different pathological populations, more and more professionals have been advocating its use as a treatment strategy in stroke survivors and the elderly. As a consequence, exercise training has recently been integrated into rehabilitation programs, both on an individual and group basis (Spain et al., 2012).

\section{Methods}

The randomized controlled study was a prospective, assessor blinded, parallel group. Potential

Corresponding Author: Moshira H. Darwish, Department of Physical Therapy for Neuromuscular Disorders, Faculty of Physical therapy, Cairo University 
participants were recruited via the Alkasr El-Ainy Multiple Sclerosis Center's computerized database.

Forty MS patients from both sex participated in this study, Their Age ranged from 25-45 years old, Last attack occured at least "three" months ago, Duration of illness started " one" to "two" years ago, Duration of immune regulatory therapy " one" to "two" years, Patients with sufficient cognitive abilities that enables them to understand and follow instructions (Mini-Mental Scale >24), Ambulant patients with EDSS of each patient ranged between 1.0 and 4.5, the treatment were conducted two session per week for successive two months, Pre and post assessment were done for each study and control group.

The patient were excluded if they had one of the following, any other neurological deficits or orthopedics abnormalities such as contracture or deformities, Any other inflammatory or infectious diseases, in the previous month e.g. arthritis, Pregnant patients, Cardiovascular and Pulmonary diseases, Patients were in acute relapse stage, Patients with any psychiatric disease. Outcome measures were collected twice, at initiation of the intervention programs and at termination of the 8week intervention period.

\section{Intervention programs}

The physical therapy program is comprised 16 hour session individualized training sessions, delivered over 8 weeks, plus an individualized 30-minute daily home exercise.

The program started with stretching exercises for shortened muscles(planter flexors, knee flexors, hip adductors) and to correct mal-alignment then facilitation exercises for weak muscles Through local facilitatory techniques (extroceptors, muscle tapping, vibration, muscle activation (isometric, eccentric and concentric contraction), and synkinetic stimulation for (hip flexors, hip abductors, knee flexors, ankle dorsiflexors) (Motl et al ., 2010).

Strengthening exercises for muscles have grade "three" or above in power. It was repeated according to ability of each patient .proprioceptive neuromuscular facilitation was also part of training sessions "Flexion, abduction, internal rotation with flexed knee" pattern for lower extremity. The started position was extension, adduction, and internal rotation.pelvic tilting exercises from different positions such as Anterior and posterior pelvic tilting from sitting, from sitting position on plinth, feet of the patient rested on the ground. Hips and knees flexed 90 degrees, trunk on erect position. Upper limbs extended and rested on the front of the patient on a stool or plinth at the shoulder level. The pelvic rested almost on posterior pelvic tilting. The therapist stood behind the patient at the level of the pelvic. Both thumbs of the therapist put on the posterior superior iliac spine to guide the movement, and the other fingers fanning at the gluteus medius muscles for its activation. Then the patient was asked to tilt pelvis anteriorly. Then return to the starting position (Moe-Nilssen, $1998 \mathrm{a}, \mathrm{b}$ ).

Lateral pelvic tilting from sitting position, from the same position, therapist stood behind the patient with both hands of the therapist fanning at the gluteus medius muscle to activate the hip abductors, and to assess weight shifting and weight acceptance a on the affected side The patient was asked to move slightly in the anterior pelvic tilt. While the therapist squeezing by one hand the gluteus medius muscle on the side on which the patient accept the weight on it, The other hand of the therapist shifting the weight diagonally to that side in the lateral direction (Rietberg et al., 2004).

Sit to stand exercises, from erect sitting position on a chair without back support with an average height. Both hands of the therapist rested on the anterior aspect of thigh. The affected foot rested backward to achieve more weight bearing on it. Sit to stand exercise, Patient started firstly by performing anterior pelvic tilting. Then, trunk extension followed by hip flexion and transition of weight on both lower limbs. Then, Lifting off from the chair by performing hip extension, knee extension and lastly knee extension (Gouelle et al., 2011).

Pelvic rotation on a therapeutic ball, from supine lying position, both hips and knees flexed 90 。 degree and rested on a therapeutic ball. The therapist put one hand on the abdominal muscles to activate and to assess the abdominal contraction in the different positions. The other hand of the therapist for and saving. The patient was asked to segmentally rotate and tilt both knees slowly to the left and right sides as far as possible (segmental rotation).gait training with biodex gait trainer with feedback for speed and step length and other gait cycles variables Exercises progressed in response to the abilities of the individual (Kjolhede et al., 2011).

Each participant received written and diagrammatic instructions describing their 30-minute daily home exercise program. The treadmill training was carried out without bodyweight support, but 
the participants were allowed to use the handrails for balance support. The training consisted of three different walking bouts, each lasting for 7 minutes: 1) preferred walking speed at an increased slope; 2) walking with verbal guidance on gait pattern, focusing on heel strike, loading and toe-off and knee control during weight-bearing; and 3) fast gait speed defined as a $10 \%$ increase in walking speed relative to preferred speed. During the 8-week intervention, walking speed in bout 3 was gradually increased with $10 \%$ to $40 \%$ of preferred speed. Preferred walking speed was used as departure point for each training session to calculate the intensity on increased velocity and inclined walking. The participants wore a heart rate monitor and were instructed to keep their exercise intensity below $70 \%$ of maximal heart rate that was obtained during the treadmill test, to keep focus on walking pattern and avoid exhaustion during gait. Each session lasted for 15 minutes, including2-minute break between the bouts (Hornby et al., 2011).

\section{Outcome measurements}

Spatio-temporal variables were obtained using biodex gait trainer Treadmill 2(zebris ${ }^{\circledR}$ Medical $\mathrm{GmbH}$, Germany). The system consists of a computer controlled treadmill fitted with more than 10,000 miniature force sensors. As the subject stands/walks on the treadmill, the instantaneous force exerted by their feet (the so-called reactive-normal force) activates the sensors. Simultaneously, targeted software utilizes special algorithms to automatically group the activated sensors and form footprints. The system integrates all footprints and provides spatio-temporal parameters as well as graphic presentation of center of pressure trajectories within static stance and gait cycle.gait speed and step length were the variables measured during this study. Data was initially examined for normality violations, outliers, errors and missing values (Henriksen et al., 2004).

\section{Data analyses}

All statistical measures were performed using the Statistical Package for Social science (SPSS) program version 23 for windows. Prior to final analysis, data were screened for normality assumption, homogeneity of variance, and presence of extreme scores. This exploration was done as a prerequisite for parametric calculations of the analysis of difference. There was a linear relationship between the dependent variables, as assessed by scatterplot, and no evidence of multicollinearity, as assessed by Pearson correlation $(|\mathrm{r}|<0.9)$. There were no univariate outliers in the data, as assessed by inspection of a boxplot, and no multivariate outliers in the data, as assessed by Mahalanobis distance. Left and right step length and speed were normally distributed, as assessed by Shapiro-Wilk's test ( $p>$ $.05)$. There was homogeneity of variances, as assessed by Levene's $(p>0.05)$ for all dependent variables. So, $2 \times 2$ mixed design MANOVA was used to compare the tested variables of interest at different tested groups and measuring periods. The alpha level was set at 0.05 .

\section{Results}

A total of 40 participants were included in the final data analysis. They were divided into two groups; group A consisted of 20 patients receiving physical therapy programin addition to treadmill training and the group B consisted of 20 patients receiving physical therapy sessions. The independent $t$ test revealed that there were no significant differences $(p>0.05)$ in the mean values of age, weight, height and BMI between both tested groups (table 1).

Table 1: Demographic characteristics of patients in both groups

\begin{tabular}{lllll}
\hline \multirow{2}{*}{ Items } & \multicolumn{1}{c}{ Group A } & \multicolumn{2}{c}{ Group B } & \multicolumn{2}{c}{ Comparison } \\
\cline { 2 - 5 } & \multicolumn{1}{c}{ Mean \pm SD } & Mean \pm SD & t-value & P-value \\
\hline Age $($ years) & $36.35 \pm 5.21$ & $35.65 \pm 4.88$ & 0.438 & 0.664 \\
Body mass (kg) & $79.95 \pm 9.12$ & $81.05 \pm 8.82$ & -0.388 & 0.701 \\
Height $(\mathbf{c m})$ & $173.3 \pm 8.41$ & $173.25 \pm 8.01$ & 0.019 & 0.985 \\
BMI $\left(\mathbf{k g} / \mathbf{m}^{\mathbf{2}}\right)$ & $30.1 \pm 2.92$ & $30.07 \pm 2.78$ & 0.035 & 0.972 \\
\hline
\end{tabular}

*SD: Standard deviation, P: Probability, S:* significance, NS: Non-significant.

Statistical analysis using mixed design MANOVA analyzed thirty patients assigned into two equal groups. It revealed that there were significant within subject effect $(F=62.832, p=0.0001)$ and treatment*time effect $(\mathrm{F}=5.742, \mathrm{p}=0.003)$. While there was not significant between subject effect $(\mathrm{F}=1.338, \mathrm{p}=0.277)$. Table $(2)$ present descriptive statistic (mean $\pm \mathrm{SD})$ and multiple pairwise 
comparison tests (Post hoc tests) for the all dependent variables. In the same context regarding within subject effect, the multiple pairwise comparison tests revealed that there was significant increase ( $p$ $<0.05$ ) in left and right step length and speed in the post treatment condition compared with the pre treatment in both groups. Regarding between subject effects multiple pairwise comparisons revealed that there was significant increase $(p<0.05)$ in speed in favor to group A than group B. while there wre no significant differences $(\mathrm{p}>0.05)$ between both groups in left and right step length.

Table 2: Descriptive statistics and Multiple pairwise comparison tests (Post hoc tests) for the all dependent variables for both groups at different measuring periods.

\begin{tabular}{lccc} 
& \multicolumn{3}{c}{ Left side } \\
\cline { 2 - 4 } Step length In (m) & $\begin{array}{c}\text { Pre treatment } \\
\text { Mean } \pm \text { SD }\end{array}$ & $\begin{array}{c}\text { Post treatment } \\
\text { Mean } \pm \text { SD }\end{array}$ & P-value \\
\hline Group A (GA) & $0.54 \pm 0.14$ & $0.61 \pm 0.16$ & $0.0001^{*}$ \\
Group B (GB) & $0.49 \pm 0.1$ & $0.53 \pm 0.1$ & $0.001^{*}$ \\
P-value & 0.21 & 0.062 & \\
\hline
\end{tabular}

*Significant: ${ }^{*} \mathrm{p}<0.05$

\begin{tabular}{cccc}
\hline Step length In (m) & $\begin{array}{c}\text { Right side } \\
\text { Pre treatment } \\
\text { Mean } \pm \text { SD }\end{array}$ & $\begin{array}{c}\text { Post treatment } \\
\text { Mean } \pm \text { SD }\end{array}$ & P-value \\
\hline Group A (GA) & $0.52 \pm 0.15$ & $0.59 \pm 0.16$ & $0.0001^{*}$ \\
Group B (GB) & $0.49 \pm 0.11$ & $0.52 \pm 0.11$ & $0.004^{*}$ \\
P-value & 0.441 & 0.164 & \\
\hline *Significant: *p<0.05 & & & P-value \\
\hline Average walking & Pre treatment & Post treatment & $<0.0001^{*}$ \\
speed(m/sec) & Mean \pm SD & Mean \pm SD & $<0.0001^{*}$ \\
\hline group A(GA) & $0.43 \pm 0.07$ & $0.53 \pm 0.07$ & $0.49 \pm 0.05$ \\
Group B (GB) & $0.43 \pm 0.05$ & 0.041 & \\
P-value & 0.99 & & \\
\hline
\end{tabular}

*Significant $: * p<0.05$

\section{Discussion}

Our main findings indicated that a 8-week physical therapy program in addition to treadmill training can improve mobility functions in people with multiple sclerosis. However, the improvements did not differ from those achieved through physical therapy program alone. There was significant increase $(p<0.05)$ in left and right step length and speed in the post treatment condition compared with the pre treatment in both groups. An interesting issue concerns the frequency of the physical activity programs, In the current trial, we demonstrated that improvements in walking in people with multiple sclerosis can be achieved in intervention programs consisting of a single weekly session (Aaslund et al., 2013).

However, single exercise sessions are limited in their ability to change walking unless they are reinforced by additional training at home. We speculate that patients who agreed to participate in the present trial (knowing that it involves exercise programs) had a positive perspective of physical activity. Consequently, compliance for the home exercise drills was probably high, contributing to the positive results. We are aware that promoting exercise in the MS community is challenging and in many cases, depression and different cognitive aspects are barriers to physical activity (Bouten et al., 1997).

An additional explanation for the similarities observed between the groups relate to the study design. A major portion of both intervention programs included the patient's daily self-practicing drills at home. In this situation, we were unable to determine if the patients had performed the exercises precisely and consistently. We sense that several participants unintentionally practiced more (or less) than others, modified several of the exercises or even trained using other exercises than those requested by the physiotherapists (Martin et al., 2006).

This possibility could have diminished the differences between the two exercise groups. 
Regarding between subject effects multiple pairwise comparisons revealed that there was significant increase $(p<0.05)$ in speed in favor to group A than group B. while there wre no significant differences $(p>0.05)$ between both groups in left and right step length.the significant increase in speed in group A can be discussed as the graduation of speed in treadmill group may have effect in increase speed in this group than group B.

Our study has limitations. Firstly, the small sample size may have influenced certain variables and influenced the results. Secondly, due to the absence of a follow-up after completion of physical therapy programs, the durability of the effect of the intervention could not be determined. Hence, further studies, including a long-term follow-up assessment, are needed to evaluate the long-term benefits of treadmill training. Finally, although we carefully followed up with each patient during the intervention program, we could not ensure home exercise compliance (Schwid et al., 2009).

There are a few considerations regarding this study. First, we do not know whether there was a lasting effect on walking in the treadmill group. Further, it would have been interesting to know whether the improvement in walking was reflected in an increase in daily activities and participation. Our study sample comprised persons with mild to moderate disease progression. Accordingly, the results may not be generalized to more severely affected persons. Also, the treadmill programme was fairly rigorous, and although it was well tolerated in the current study sample, it might not be feasible for more severely affected patients (Thoumie et al., 2005)

In conclusion, this study presents an alternative rehabilitation program for people with multiple sclerosis. While to date no prospective randomized studies using this selected program for walking rehabilitation in multiple sclerosis appear in the literature, this study shows that treadmill training is a potential treatment option for people with multiple sclerosis to improve their walking and balance capabilities. However, this approach does not have any significant advantage over physical therapy programs alone (Webster et al., 2005).

\section{References}

American College of Sports Medicine position stand, 2009. Progression models in resistance training for healthy adults. Medicine and Science in Sports and Exercise, 41:687-708.

Aaslund, M.K., J.L. Helbostad and R. Moe-Nilssen, 2013. Walking during body-weight-supported treadmill training and acute responses to varying walking speed and bodyweight support in ambulatory patients post-stroke. Physiotherapy Theory and Practice, 29: 278-289.

Bouten, C.V., A.A. Sauren, M. Verduin and J.D. Janssen, 1997. Effects of placement and orientation of body-fixed accelerometers on the assessment of energy expenditure during walking. Medical and Biological Engineering and Computing, 35: 50-56.

Cameron, M.H. and J.M. Wagner, 2011. Gait abnormalities in multiple sclerosis: Pathogenesis, evaluation, and advances in treatment. Current Neurology and Neuroscience Reports, 11: 507515.

Gouelle, A., F. Megrot, A. Presedo, G.F. Pennecot and A.Yelnik, 2011. Validity of Functional Ambulation Performance Score for the evaluation of spatiotemporal parameters ofchildren's gait. Journal of Motor Behavior, 43:95-100.

Henriksen, M., H. Lund, R. Moe-Nilssen, H. Bliddal and B. Danneskiod-Samsøe, 2004. Test-retest reliability of trunk accelerometric gait analysis. Gait \& Posture 2004; 19(3): 288-297.

Hornby, T.G., D.S. Straube, C.R. Kinnaird, C.L. Holleran, A.J. Echauz, K.S. Rodriguez, E.J. Wagner and E.A. Narducci, 2011. Importance of specificity, amount, and intensity of locomotor training to improve ambulatory function in patients poststroke. Topics in Stroke Rehabilitation, 18(4): 293-307.

Kjolhede, T., K. Vissing and U. Dalgas, 2012. Multiple sclerosis and progressive resistance training: A systematic review. Multiple Sclerosis, 18(9): 1215-1228.

Martin, C.L., B.A. Phillips, T.J. Kilpatrick, H. Butzkueven, N. Tubridy, E. Mcdonald and M.P. Galea, 2006. Gait and balance impairment in early multiple sclerosis in the absence of clinical disability. Multiple Sclerosis, 12: 620-628. 
Moe-Nilssen, R.,1998a. A new method for evaluating motor control in gait under real-life environmental conditions. Part 1: The instrument. Clinical Biomechanics (Bristol, Avon), 13: 320-327.

Moe-Nilssen, R., 1998b. A new method for evaluating motor control in gait under real-life environmental conditions. Part 2: Gait analysis. Clinical Biomechanics (Bristol, Avon), 13: 328-335.

Motl, R.W., Goldman MD, Benedict RH. Walking impairment in patients with multiple sclerosis: exercise training as a treatment option. Neuropsychiatric Disease and Treatment 2010; 6: 767774.

Rietberg, M.B., D. Brooks, B.M.J. Uitdehaag and G.Kwakkel,2004 Exercise therapy formultiple sclerosis. Cochrane Database of Systematic Reviews.

Schwid, S.R., C.A. Thornton, S. Pandya, K.L. Manzur, M. Sanjak, M.D. Petrie, M.P. Mcdermott and A.D. Goodman, 1999. Quantitative assessment of motor fatigue and strength in MS. Neurology, 53: 743-750.

Spain, R.I., R.J. St George, A. Salarian, M. Mancini, J.M. Wagner, F.B. Horak and D. Bourdette, 2012. Body-worn motion sensors detect balance and gait deficits in people with multiple sclerosis who have normal walking speed. Gait \& Posture, 35: 573-578.

Thoumie, P., D. Lamotte, S. Cantalloube, M. Faucher and G. Amarenco, 2005. Motor determinants of gait in 100 ambulatory patients with multiple sclerosis. Multiple Sclerosis, 11: 485-491.

Webster, K.E., J.E. Wittwer and J.A. Feller, 2005. Validity of the GAITRite walkway system for the measurement of averaged and individual step parameters of gait. Gait \& Posture, 22: 317-321. 